\title{
Polarization gating in ultrafast-optics imaging of skeletal muscle tissues
}

\author{
Chia-Wei Sun \\ Department of Electrical Engineering and Graduate Institute of Electro-Optical Engineering, \\ National Taiwan University, Taipei, Taiwan \\ Chih-Yu Wang \\ Department of Bioengineering, I-Shou University, Kaohsiung, Taiwan
}

C. C. Yang, Yean-Woei Kiang, and I-Jen Hsu

Department of Electrical Engineering, Graduate Institute of Electro-Optical Engineering and Graduate Institute of Communication Engineering, National Taiwan University, 1, Roosevelt Road, Sec. 4, Taipei 107, Taiwan

\section{Chii-Wann Lin}

Graduate Institute of Bioengineering, National Taiwan University, Taipei, Taiwan

\author{
Received September 13, 2000
}

\begin{abstract}
By comparing the results of polarization-dependent, time-resolved intensity profiles of photons transmitted through diluted milk, chicken breast tissue, and chopped chicken breast tissue, we found that the inherent anisotropic optical property of skeletal muscle tissue resulted in coherent coupling between two mutually perpendicular polarization directions. This coupling process led to difficulty in using the conventional polarization gating method for imaging unless the anisotropy characteristics were well understood. However, imaging based on polarization gating in diluted milk and chopped chicken breast tissue, which had an isotropic random-scattering nature, was quite effective. () 2001 Optical Society of America

OCIS codes: $170.0170,170.3880,170.3660$.
\end{abstract}

Ultrafast optics has been widely used for optical imaging of human tissues based on time gating of transmitted photons. In such a technique, a short pulse (picosecond to femtosecond) is applied to tissues, and the transmitted snake photons are extracted with various time-gating techniques, ${ }^{1,2}$ including the use of a streak camera. ${ }^{3}$ Besides time gating, polarization gating is another important method in biomedical optical imaging. This method is based on the depolarization effect in random scattering of tissues. Hence, with linearly polarized input signals, the copolarized transmitted photons will be more coherent and carry more information on tissue structures. Subtraction of the cross-polarized component from the copolarized component, i.e., the removal of the incoherent part, has been shown to be useful for effective optical imaging. ${ }^{4,5}$

Although phantoms such as intralipid and milk have been widely used for imaging studies, recently attention was paid to the differences in scattering characteristics between real tissues and phantoms. ${ }^{6,7}$ In this Letter we report the results of ultrafast imaging of skeletal muscle tissues, based on the polarization-gating technique. Because such tissues consist of filaments with optically isotropic $I$ bands (actin) and anisotropic $A$ bands (myosin), as reported, e.g., in Ref. 8, their optical transmission characteristics are quite different from those of other kinds of tissue and of widely used phantoms. ${ }^{9}$ We compared time-gated results from chicken breast tissue, chopped chicken breast tissue, and diluted milk (phantom) used to demonstrate the anisotropic characteristics of skeletal muscle tissues.

In the experiment (with the setup shown in Fig. 1), an argon-laser-pumped mode-locked Ti:sapphire laser was used to provide $\sim 100$-fs laser pulses at $800 \mathrm{~nm}$. The laser beam was split into two branches, one for propagating through samples and the other for temporal reference. Two polarizers, one before and the other after the samples, were used to control the input and output polarization for polarization gating. After recombination of the sample and reference beams, the signals were directed to a streak camera with a

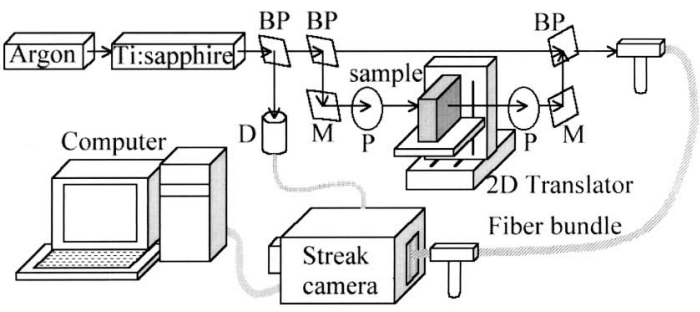

Fig. 1. Experimental setup: BP's, beam splitters; P's, polarizers; M's, mirrors; D, detector; 2D, two-dimensional. 
fiber bundle. The temporal resolution of the operation mode of the streak camera was $4.7 \mathrm{ps}$.

Figure 2 shows the time-resolved intensity profiles of three cases. Cases 1,2 , and $3(x=1,2,3)$ describe the results for diluted milk, chicken breast tissue, and chopped chicken breast tissue, respectively. In each case, curves $I_{\mathrm{p} x}$ and $I_{\mathrm{c} x}(x=1,2,3)$ represent the copolarized and the cross-polarized output components, respectively. The diluted milk was contained in a plastic vessel with a transmission length of $10 \mathrm{~cm}$. The chicken breast tissue sample varied in thickness from 1.3 to $1.5 \mathrm{~cm}$ within the transverse scanning area. The transmission length of chopped chicken breast tissue was $1.4 \mathrm{~cm}$. It was estimated that the size of the chopped chicken pieces was in the millimeter range. The scanning mode was used in the measurements. The scanning pixel size was $1 \mathrm{~mm} \times 1 \mathrm{~mm}$, and the scanning area was $2 \mathrm{~cm} \times 2 \mathrm{~cm}$. We obtained the results shown in Fig. 2 by averaging over all the scanning pixels (a total of 400 pixels). Also, all the intensity curves were normalized and synchronized so that the peaks of the three $I_{\mathrm{p}}$ profiles had unity intensity and were located at the same time. With this procedure, one can still observe a relatively shorter scattering tail of the chicken tissue compared with that of the other two cases. This difference can be attributed to the organized tissue structure in skeletal muscles. Such structure was destroyed (at least partly) in the case of the chopped chicken tissue. In that case, random scattering became stronger, and hence the intensity peak was delayed and the scattering tail was elongated.

In Fig. 2, comparisons of intensity and temporal peak position within the same case can also provide us with important information. In the case of diluted milk, $I_{\mathrm{c}}$ is clearly lower than $I_{\mathrm{p}}$. Also, the peak of $I_{\mathrm{c}}$ is delayed by that of $I_{\mathrm{p}}$. These observations represent typical results of isotropic scattering, in which diffuse photons are equally distributed in the two mutually perpendicular polarization directions after strong scattering. In this situation, subtraction of $I_{\mathrm{c}}$ from $I_{\mathrm{p}}$ results in coherent photons for effective imaging. Figure 3 shows an example. In the figure, two pieces of lean pork (2 and $1 \mathrm{~mm}$ in thickness) were placed in diluted milk for imaging. Figure 3(a) shows an image of the integrated intensity with appropriate time gating of $I_{\mathrm{p}}$. Figure 3(b) shows the image of time-gated $I_{\mathrm{p}}-I_{\mathrm{c}}$. Although $I_{\mathrm{p}}$ can show the locations (dark spots) of the two pieces of pork, $I_{\mathrm{p}}-I_{\mathrm{c}}$ provides a clearer image, particularly for the smaller and thinner piece on the right.

In the case of chicken breast tissue (case 2), the intensity profiles in Fig. 2 show that $I_{\mathrm{c}}$ is slightly higher than $I_{\mathrm{p}}$. This result indicates that, besides random scattering in such a medium, coherent coupling between the two polarization components occurs as a result of the anisotropic characteristics of the tissue. Although the input linear polarization was close to the direction of the tissue filaments, signal polarization seemed to rotate such that $I_{\mathrm{c}}$ became higher than $I_{\mathrm{p}}$ at the output. In this situation, both $I_{\mathrm{p}}$ and $I_{\mathrm{c}}$ were quite coherent, particularly before and near their peaks. Hence, the conventional polarization gating technique, i.e., imaging with $I_{\mathrm{p}}-I_{\mathrm{c}}$, became difficult. Figure 4 shows imaging results with a thin chicken bone (diameter, 1-2 $\mathrm{mm}$ ) sticking into the breast tissue sample. The result for $I_{\mathrm{p}}$ provides a rough picture of the chicken bone; however, $I_{\mathrm{p}}-I_{\mathrm{c}}$ shows no features at all. The imaging results confirm the

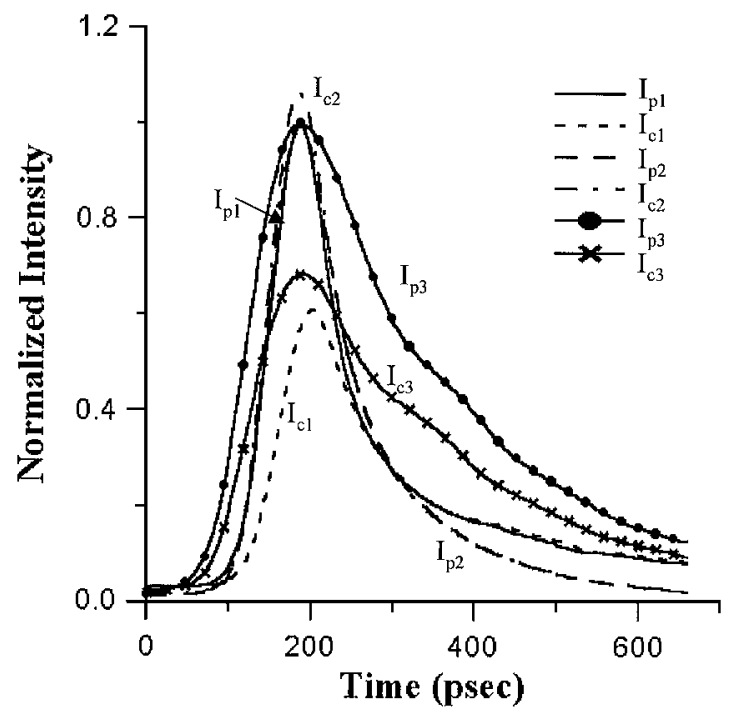

Fig. 2. Normalized and synchronized intensity profiles of cases 1,2 , and 3, corresponding to samples of diluted milk, chicken breast tissue, and chopped chicken breast tissue. $I_{\mathrm{p} x}$ and $I_{\mathrm{c} x}$ represent the intensities of the copolarized and cross-polarized components, respectively, for case $x$ $(x=1,2,3)$.
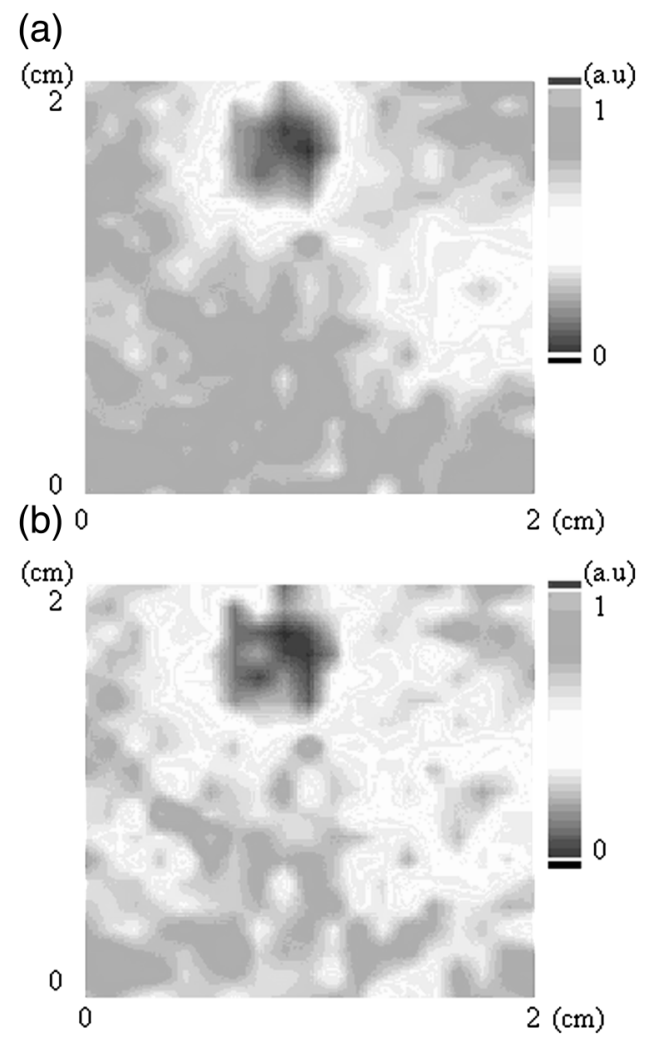

Fig. 3. Time-gated images of two pieces of lean pork in diluted milk with (a) $I_{\mathrm{p}}$ and (b) $I_{\mathrm{p}}-I_{\mathrm{c}}$. 
(a)

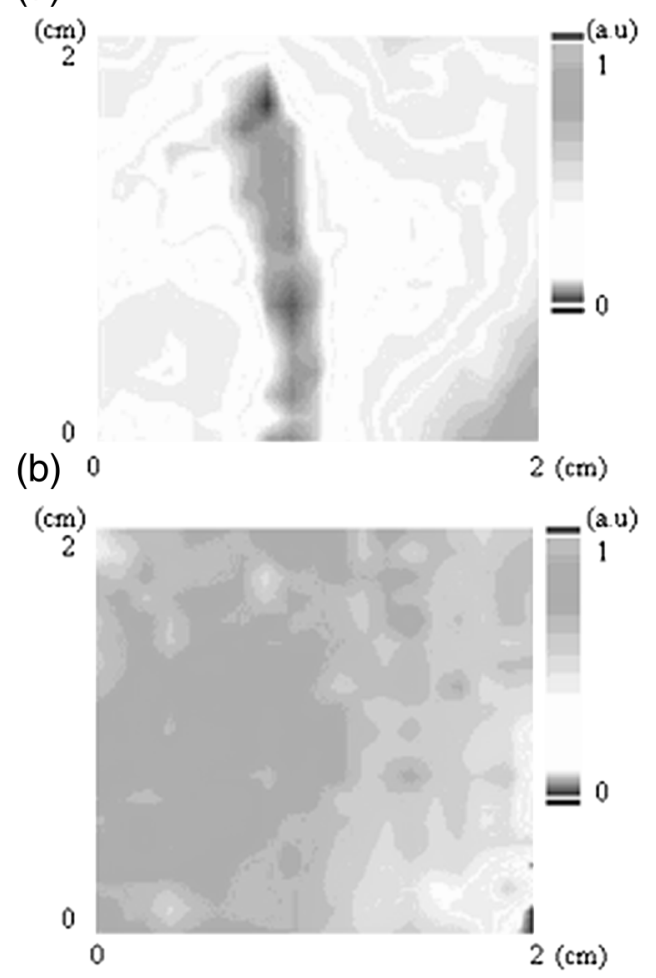

Fig. 4. Time-gated images of a chicken bone in chicken breast tissue with (a) $I_{\mathrm{p}}$ and (b) $I_{\mathrm{p}}-I_{\mathrm{c}}$.

coherent polarization evolution besides random scattering in skeletal muscle tissues.

Then, in the case of chopped tissue, we chopped and stirred the chicken tissue to make it statistically homogeneous. However, because the chopped pieces still had a size in the millimeter range, the basic tissue structures might still have been preserved. In this situation, as shown in Fig. $2, I_{\mathrm{p}}$ is significantly stronger than $I_{\mathrm{c}}$, with almost no time difference between their peaks. The relatively broader intensity profiles of chopped chicken tissue than those of the chicken tissue indicate stronger random scattering in the former. Figure 5 shows images of $I_{\mathrm{p}}$ and $I_{\mathrm{p}}-I_{\mathrm{c}}$ with the same chicken bone sticking into the chopped chicken tissue. A rough picture of the bone can be seen from $I_{\mathrm{p}}$. The detailed configuration of the bone can be seen from $I_{\mathrm{p}}-I_{\mathrm{c}}$. The results in Figs. 2 and 5 reveal the mixed effects of coherent polarization coupling and enhanced random scattering in chopped chicken tissue.

This research was supported by the National Health Research Institute of the Republic of China under grant NHRI-GT-EX89. C. C. Yang's e-mail address is ccy@cc.ee.ntu.edu.tw.

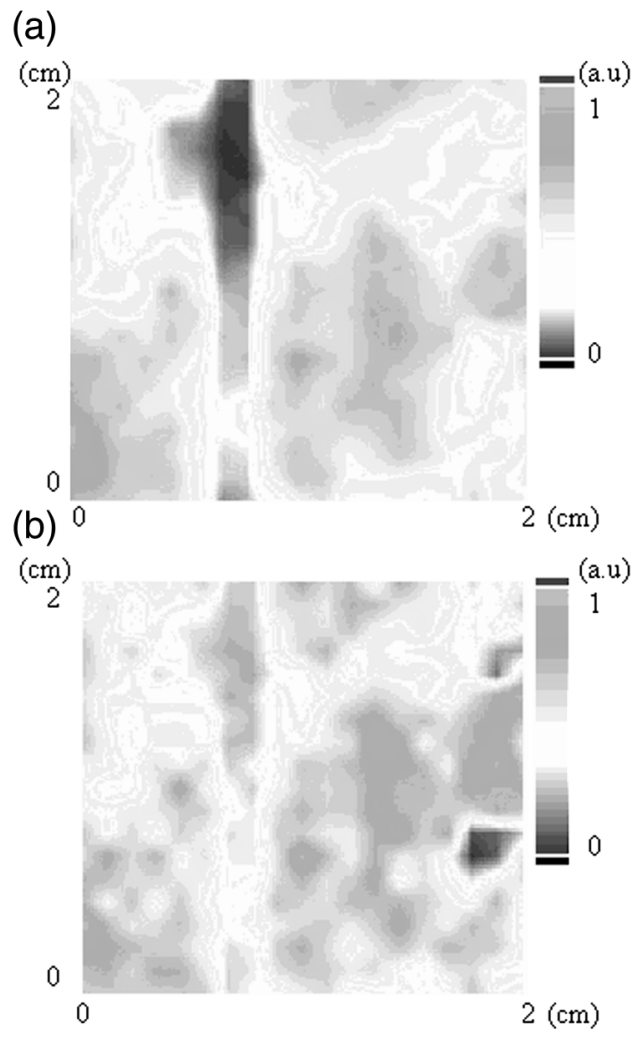

Fig. 5. Time-gated images of a chicken bone in chopped chicken breast tissue with (a) $I_{\mathrm{p}}$ and (b) $I_{\mathrm{p}}-I_{\mathrm{c}}$.

\section{References}

1. L. M. Wang, P. P. Ho, and R. R. Alfano, Appl. Opt. 32, 535 (1993).

2. G. W. Faris and M. Banks, in Advances in Optical Imaging and Photon Migration, Vol. 21 of OSA Proceedings Series (Optical Society of America, Washington, D.C., 1994), p. 139.

3. K. M. Yoo, B. B. Das, and R. R. Alfano, Opt. Lett. 17, 958 (1992).

4. H. Horinaka, K. Hashimoto, K. Wada, Y. Cho, and M. Osawa, Opt. Lett. 20, 1501 (1995).

5. S. G. Demos and R. R. Alfano, Opt. Lett. 21, 161 (1996).

6. V. Sankaran, M. J. Everett, D. J. Maitland, and J. T. Walsh, Jr., Opt. Lett. 24, 1044 (1999).

7. V. Sankaran, J. T. Walsh, Jr., and D. J. Maitland, Opt. Lett. 25, 239 (2000).

8. A. J. Vander, J. H. Sherman, and D. S. Luciano, Human Physiology: The Mechanisms of Body Function, 6th ed. (McGraw-Hill, New York, 1994), p. 304.

9. G. Marquez, L. V. Wang, S. P. Lin, J. A. Schwartz, and S. L. Thomsen, Appl. Opt. 37, 798 (1998). 